\title{
Inhibition of the Wnt/ $\beta$-catenin pathway enhances antitumor immunity in ovarian cancer
}

\author{
David W. Doo, Selene Meza-Perez, Angelina I. Londoño, Whitney N. Goldsberry, \\ Ashwini A. Katre, Jonathan D. Boone, Dylana J. Moore, Cindy T. Hudson, Ilaria Betella (iD) \\ Tyler R. McCaw, Abhishek Gangrade, Riyue Bao, Jason J. Luke, Eddy S. Yang, \\ Michael J. Birrer, Dmytro Starenki, Sara J. Cooper, Donald J. Buchsbaum, \\ Lyse A. Norian, Troy D. Randall and Rebecca C. Arend
}

\begin{abstract}
Background: The $\mathrm{Wnt} / \beta$-catenin pathway is linked to tumorigenesis in a variety of tumors and promotes $T$ cell exclusion and resistance to checkpoint inhibitors. We sought to determine whether a small molecule inhibitor of this pathway, WNT974, would impair tumor growth, affect gene expression patterns, and improve the immune response in human and murine ovarian cancer models.

Methods: Human ovarian cancer cells were treated with WNT974 in vitro. RNAseq libraries were constructed and differences in gene expression patterns between responders and nonresponders were compared to The Cancer Genome Atlas (TCGA). Mice with subcutaneous or intraperitoneal ID8 ovarian cancer tumors were treated with WNT974, paclitaxel, combination, or control. Tumor growth and survival were measured. Flow cytometry and $\beta$ TCR repertoire analysis were used to determine the immune response.

Results: Gene expression profiling revealed distinct signatures in responders and nonresponders, which strongly correlated with T cell infiltration patterns in the TCGA analysis of ovarian cancer. WNT974 inhibited tumor growth, prevented ascites formation, and prolonged survival in mouse models. WNT974 increased the ratio of $\mathrm{CD} 8^{+} \mathrm{T}$ cells to T regulatory cells (Tregs) in tumors and enhanced the effector functions of infiltrating $\mathrm{CD}^{+}$and $\mathrm{CD} 8^{+} \mathrm{T}$ cells. Treatment also decreased the expression of inhibitory receptors on $\mathrm{CD}^{+} \mathrm{T}$ cells. Combining WNT974 with paclitaxel further reduced tumor growth, prolonged survival, and expanded the T cell repertoire.

Conclusions: These findings suggest that inhibiting the $\mathrm{Wnt} / \beta$-catenin pathway may have a potent immunomodulatory effect in the treatment of ovarian cancer, particularly when combined with paclitaxel.
\end{abstract}

Keywords: cancer immunology, immune checkpoints, immunotherapy, ovarian cancer, paclitaxel, tumor immunity, Wnt/ $\beta$-catenin pathway

Received: 30 August 2019; revised manuscript accepted: 26 February 2020.

\section{Introduction}

Ovarian cancer is the most deadly gynecologic malignancy, with over 14,000 deaths estimated to occur in 2018 in the USA. ${ }^{1}$ Despite advances in surgery and chemotherapy, mortality rates have not significantly improved and new therapeutic modalities are needed. One pathway that shows promise for therapeutic targeting is the $\mathrm{Wnt} / \beta$-catenin pathway, which in ovarian cancer contributes to a stem cell-like state, and its activity is associated with a worse overall prognosis. ${ }^{2,3}$ The $\mathrm{Wnt} / \beta$-catenin signaling pathway is normally activated during embryonic development in a variety of tissues. However, mutations in this pathway are associated with breast, colorectal, hepatocellular, and endometrial cancers. ${ }^{4}$ Mechanistically, activation of the
Ther Adv Med Oncol 2020, Vol. 12: 1-16 DOI: $10.1177 /$ 1758835920913798

(c) The Author(s), 2020. Article reuse guidelines: sagepub.com/journalspermissions

Correspondence to: Rebecca C. Arend Department of Obstetrics and Gynecology, Division of Gynecologic Oncology, University of Alabama at Birmingham, 619 19th Street South, 176F Rm 10250, Birmingham, AL 35249, USA

Comprehensive Cancer Center, University of Alabama at Birmingham, $A L$, USA rarendauabmc.edu

David W. Doo

Angelina I. Londoño Whitney N. Goldsberry Ashwini A. Katre Jonathan D. Boone

Dylana J. Moore Cindy T. Hudson Department of Obstetrics and Gynecology, Division of Gynecologic Oncology, University of Alabama at Birmingham, Birmingham, $A L$, USA

Selene Meza-Perez

Tyler R. McCaw

Department of

Medicine, Division of

Clinical Immunology and Rheumatology, University of Alabama at Birmingham, Birmingham, AL, USA

Ilaria Betella

Comprehensive Cancer Center, University of Alabama at Birmingham,

AL, USA

Abhishek Gangrade Department of Radiation Oncology, University of Alabama at Birmingham, Birmingham, AL, USA

Riyue Bao

Department of Pediatrics, University of Chicago School of Medicine, Chicago, IL, USA

Center for Research Informatics, University of Chicago, Chicago, IL, USA Jason J. Luke Department of Medicine, University of Chicago

School of Medicine.

Chicago, IL, USA 
Eddy S. Yang

Donald J. Buchsbaum

Comprehensive Cancer

Center, University of

Alabama at Birmingham,

$A L$, USA

Department of Radiation Oncology, University of

Alabama at Birmingham,

Birmingham, AL, USA

Michael J. Birrer

Department of Obstetrics and Gynecology, Division

of Gynecologic Oncology.

University of Alabama at

Birmingham, Birmingham,

AL, USA

Comprehensive Cancer Center, University of

Alabama at Birmingham,

$A L$, USA

Dmytro Starenki

Sara J. Cooper

Hudson Alpha Institute for

Biotechnology, Huntsville,

AL, USA

Lyse A. Norian

Comprehensive Cancer

Center, University of

Alabama at Birmingham,

$A L, U S A$

Department of Nutrition

Sciences, University of

Alabama at Birmingham,

Birmingham, AL, USA

Troy D. Randall

Department of Medicine,

Division of Clinical

Immunology and

Rheumatology, University

of Alabama at Birmingham,

Birmingham, AL, USA

Comprehensive Cancer

Center, University of

Alabama at Birmingham,

$A L$, USA
Wnt/ $\beta$-catenin signaling pathway in tumors maintains their stem cell properties, ${ }^{5}$ regulates cell proliferation and apoptosis, ${ }^{6}$ and promotes chemoresistance by regulating the epithelial to mesenchymal transition (EMT). ${ }^{7}$

The Wnt proteins are a highly conserved family of at least 19 cysteine-rich secreted glycoproteins ${ }^{8}$ that bind to a G-protein-coupled receptor in the Frizzled family in a complex with the low-density lipoprotein receptor-related protein 6 (Lrp6). ${ }^{9} \mathrm{Wnt}$ binding to its receptor prevents $\beta$-catenin destruction in the proteasome and allows it to enter the nucleus, where it cooperates with other transcription factors to drive gene expression. ${ }^{9}$ Wnt proteins require post-translational palmitoylation or O-acylation, which enhances Wnt secretion and promotes binding to its receptor. ${ }^{10}$ Addition of palmitoyl groups to Wnt proteins is catalyzed by the membranebound, O-acyltransferase enzyme, porcupine (PORCN). ${ }^{11}$ As a result, inhibitors of PORCN can block Wnt secretion, impair Wnt signaling, and limit the growth of some tumor cells. ${ }^{12}$

In addition to its effects on tumor cells, the Wnt signaling pathway also impacts immune responses. For example, the Wnt/ $\beta$-catenin signaling pathway helps to maintain dendritic cells (DCs) in a tolerogenic state and prevents autoimmune responses. ${ }^{13}$ More recent data show that the Wnt/ $\beta$-catenin pathway suppresses antitumor immune response in melanoma by suppressing the accumulation of $\mathrm{CD}_{103}{ }^{+} \mathrm{DCs}$ and thereby preventing the activation of T cells. ${ }^{14}$ Thus, active $\mathrm{W} n \mathrm{nt} / \beta$-catenin signaling in ovarian cancer may directly promote tumor growth as well as suppress antitumor immune responses.

Here we tested whether ovarian cancer cells were sensitive to the inhibition of Wnt signaling and whether this pathway played a role in immune evasion. Using the PORCN antagonist, WNT974, we divided ovarian cancer samples into responders and nonresponders and showed that their gene expression profiles were strikingly similar to non-T-cell inflamed and T-cell inflamed (respectively) ovarian cancer samples from The Cancer Genome Atlas (TCGA). WNT974 also impaired the growth of ovarian cancer cells in syngeneic mice, prevented ascites accumulation, and prolonged survival. These changes correlated with an increase in the CD8: Treg ratio, increased cytokine production, and reduced inhibitory receptor expression by CD8 $\mathrm{T}$ cells. Wnt inhibition also correlated with an increased frequency of total
DCs in tumors, although CD103+ DCs were not significantly increased. Finally, the combination of paclitaxel and WNT974 further decreased tumor size, increased the CD8:Treg ratio, increased the number of distinct CDR3 sequences in the TCR repertoire, and prolonged survival. Thus, inhibition of the Wnt signaling pathway promotes antitumor immunity and impairs the progression of ovarian cancer.

\section{Methods}

\section{Ascites specimen collection and processing}

All patient studies were conducted in accordance with the ethical guidelines of the Belmont Report. This study was approved by the UAB Institutional Review Board for Human Use prior to human specimen collection (Approval \#IRB-131007005). Written consent for participation in this study was obtained from all patients prior to surgery. Ascites was collected from 60 patients at the time of surgical debulking for papillary serous ovarian or primary peritoneal cancer, and cells were isolated as previously described. ${ }^{15}$ Cells were stored in $10 \%$ DMSO and the viability of ascites cells was confirmed with trypan blue staining. Seven samples were excluded because there were not enough viable cells after thawing to proceed with testing.

\section{Ascites cell treatment and ATPlite}

luminescence-based assay

Patient-derived ascites cells were plated in a 96-well ultra-low attachment plate at 2000 cells/ well in $45 \mu \mathrm{l} \mathrm{X}$-vivo serum free media containing $20 \mathrm{ng} / \mathrm{ml}$ epidermal growth factor and $10 \mathrm{mg} / \mathrm{ml}$ bovine insulin (all from Corning Costar, Corning, NY, USA). They were then treated in 16 replicates with $50 \mathrm{nM}$ WNT974 (Novartis, Cambridge, MA, USA) for 7 days, lysed, and viability was analyzed using the ATPlite luminescence-based assay (PerkinElmer, Waltham, MA, USA) as previously described. ${ }^{15}$

\section{RNA sequencing}

RNA was extracted from ascites cells using the Norgen Total RNA extraction kit (cat. \# 17200, Norgen, Thorold, ON, Canada). Input was normalized based on cell count. RNA integrity numbers were measured using BioAnalyzer (Agilent, Santa Clara, CA, USA) and ranged from 7.1 to 9.9 (with one outlier of 2.9 , which was removed from further analysis). RNA was quantified using 
Qubit (Thermofisher). Then, $800 \mathrm{ng}$ of total RNA was used as input to the NEBNext Ultra RNA Library Prep Kit for Illumina using the polyA selection method (E7530S and E7490S). Libraries were barcoded using NEBNext multiplex oligos for Illumina (E7335S). Five samples per lane were pooled on an Illumina HiSeq 2500 flow cell and sequenced an average of $29 \mathrm{M}$ aligned reads per sample and an average quality score of 35, with $93 \%>$ Q30. Samples were processed using our published primary analysis tool, aRNApipe. ${ }^{16}$ Count tables were processed using DESeq $2^{17}$ for differential expression analysis. No clinical covariates were used in the model since the experiment was all done in vitro. $\mathrm{R}$ was used for all additional statistical analysis. Hierarchical clustering was done using the heatmap.2 function in the gplots $\mathrm{R}$ package.

\section{TCGA analysis}

RNA-Seq gene expression data were downloaded for ovarian serous adenocarcinoma from TCGA, which were preprocessed by Broad Institute's TCGA workgroup (release date February 4, 2015). Gene expression was quantified by the RNA-Seq by Expectation Maximization (RSEM) method, ${ }^{18}$ followed by upper-quartile normalization and $\log 2$ transformation. Primary tumor samples of $261 \mathrm{OV}$ patients were categorized into T-cell inflamed $(n=86)$, intermediate $(n=106)$, and non-T-cell inflamed group $(n=69)$ using a defined $\mathrm{T}$-cell inflamed signature as previously described. ${ }^{19}$

\section{Cell lines and culture}

The ID8 murine epithelial ovarian cancer cell line was provided by Dr. Yancey Gillespie (University of Alabama at Birmingham, Birmingham, Alabama). Cells were maintained in RPMI1640 (Corning) supplemented with $10 \%$ fetal bovine serum (Atlanta Biologicals, Flowery Branch, GA, USA). Mullerian Inhibitory Substance type II Receptor (MISIIR) ovarian cancer (MOVCAR) cells (Dr. Denise Connolly, Fox Chase Cancer Center, Philadelphia, PA) were originally established from tumors of C57BL/6 TgMISIIR-TAg transgenic mice. MOVCAR cells were maintained in Dulbecco's modified eagle medium (DMEM; Corning) supplemented with $4 \%$ fetal bovine serum, 1X insulin/selenium/transferrin (Invitrogen), penicillin/streptomycin, and $2 \mathrm{mM}$ L-glutamine. Cells were passaged by dissociation with $0.025 \%$ trypsin, $0.53 \mathrm{mM}$ EDTA without sodium bicarbonate (Corning), washed, and replated. Cells were maintained in culture fewer than 10 passages from the parent stock. Experiments were performed at $70-80 \%$ confluency.

\section{Mouse studies}

Animal studies were approved by the University of Alabama at Birmingham Institutional Animal Care and Use Committee (Approval \#IACUC20205). All animal studies followed institutional, national, and international guidelines for the humane treatment of experimental animals. C57BL/6 mice and NCG mice (NOD CRISPR Prkdc il2r gamma), were obtained from NCI Charles River (Wilmington, MA, USA). Experiments were performed in mice at 7-8 weeks of age. For SC tumor models, C57BL/6 mice were injected in the right flank with $7 \times 10^{6}$ ID8 cells in a 1:1 mixture of phosphate-buffered saline (PBS; Corning) and growth-factor reduced Matrigel ${ }^{\circledR}$ Matrix basement membrane (Corning). Tumors were measured with calipers (length $\times$ width) twice per week, reported in $\mathrm{mm}^{2}$, and treatment was started 21 days after cell injection. For intraperitoneal (IP) tumor models, C57BL/6 and NCG mice were injected with $7 \times 10^{6}$ ID 8 cells in $200 \mu \mathrm{l}$ PBS and treatment was started 28 days after cell injection.

MISIIR-TAg-Low mice were obtained from Dr. Denise Connolly, which express SV40 T antigen under the MISIIR promoter and have little susceptibility to spontaneous tumor development. ${ }^{20}$ Transgene-positive male mice were bred with C57BL/6 mice and female offspring were genotyped by PCR (polymerase chain reaction) using DNA extracted from ear punches. These were tested for the heterozygous expression of the MISIIR promoter using the primers TGCA TGGTGTACAACATTCC and TTGGGACT GTGAATCAATGCC and Expand High Fidelity taq polymerase (Roche) with 30 cycles of $95^{\circ} \mathrm{C}$ for $30 \mathrm{~s}, 60^{\circ} \mathrm{C}$ for $30 \mathrm{~s}$, and $72^{\circ} \mathrm{C}$ for $30 \mathrm{~s}$. PCR products were analyzed by agarose gel electrophoresis and a band of $773 \mathrm{bp}$ indicated the presence of the MISIIR transgene. Transgene-positive females were injected intraperitoneally with $7 \times 10^{6}$ MOVCAR cells line derived from the MISIIR ovarian cancer (MOVCAR) cell line in $200 \mu \mathrm{l}$ PBS, and treatment was started 28 days after cell injection.

Mice with either subcutaneous (SC) or IP tumors were randomized to treatment with vehicle 
control, paclitaxel, WNT974, or combination of paclitaxel and WNT974. Paclitaxel (Pfizer, New York, NY, USA) was diluted in PBS and administered by IP injection at a dose of $5 \mathrm{mg} / \mathrm{kg}$ in a schedule of 3 days on/ 3 days off for a total of nine doses in order to mimic a dose-dense administration strategy. ${ }^{21}$ WNT974 was dissolved in $0.5 \%$ Tween 80 (Thermofisher) and $0.5 \%$ methylcellulose (Thermofisher) in ddH20 and administered by oral gavage at $5 \mathrm{mg} / \mathrm{kg}$ twice daily for 1 week, followed by $2.5 \mathrm{mg} / \mathrm{kg}$ twice daily for 1 week, when treatment was given for 14 days. When treatment was given for 28 days, the same 14-day regimen was given followed by $2.5 \mathrm{mg} / \mathrm{kg}$ daily for 2 additional weeks. The dosage was tapered due to toxicity that was encountered in prior experiments.

Mice with SC tumors were given 4 weeks of treatment and tumor growth was measured for 40 days from the initiation of treatment, at which point the mice were sacrificed. Mice were monitored twice per week and were sacrificed if they had body condition score $\leqslant 2$, inhibition of movement by tumors, severe tumor ulceration, or became moribund per UAB IACUC Policies and Guidelines. Mice with IP tumors were sacrificed after 14 days of treatment to measure omental tumor weights, ascites volume, and to process tumors for analysis by flow cytometry, NanoString analysis, or $\mathrm{T}$ cell repertoire analysis. For survival analysis, a separate cohort of mice with IP tumors was treated for the full four-week course and then observed until they developed ascites severe enough to inhibit movement or became moribund, at which point they were sacrificed.

\section{NanoString nCounter mRNA analysis}

Omental tumors were harvested, fixed in $10 \%$ formalin for $24 \mathrm{~h}$, and put into paraffin blocks. Microtome blades treated with RNase AWAY (Life Technologies, Carlsbad, CA, USA) were used to collect ten $10 \mathrm{uM}$ sections. RNA was isolated using the Invitrogen Purelink FFPE Total RNA Isolation Kit (Life Technologies). Briefly, the paraffin was melted, resulting in visible ribbons of tissue. Proteinase $\mathrm{K}$ was added and the tissue was incubated for $2.5 \mathrm{~h}$ to obtain complete lysis. The lysate was purified according to kit protocol and eluted into two $30 \mathrm{ul}$ aliquots. Quantification was performed using the DeNovix DS-11 Spectrophotometer (Denovix Inc., Wilmington, DE, USA). Samples of high purity (A260/A280>2, A260/A230>1.4) were processed on the NanoString nCounter Flex system
(NanoString Technologies, Seattle, WA, USA) using the mouse PanCan Pathways and mouse PanCan Immune premade panels. Briefly, $100 \mathrm{ng}$ of purified RNA was hybridized for at least $16 \mathrm{~h}$ with the respective Reporter Code set and Capture Probes for each panel separately. The samples were purified and immobilized on the NanoString Prep Station and counted on the NanoString Digital Analyzer. Analysis was performed using nSolver 3.0 software (NanoString Technologies). Cluster 3.0 and Java TreeView-1.1.6r4 were used to create heat maps. Expression levels were normalized to housekeeping genes that were not discarded by the gNorm program in the advanced analysis module. T cell infiltration was also calculated using a similar approach as described above for the TCGA data; however, only 33 genes (Supplementary Table 1) were measured on the NanoString Immune panel.

\section{Flow cytometry}

Omental tumors were harvested and placed in digestion buffer (DMEM 25Mm HEPES, 3.5\% fatty acid free BSA (bovine serum albumin) plus $0.5 \mathrm{mg} / \mathrm{ml}$ collagenase $+70 \mathrm{ug} / \mathrm{ml}$ DNAse I) (all from Corning) and fragmented with scissors. Tumor fragments were placed in an orbital shaker at $250-300 \mathrm{rpm}$ for $30 \mathrm{~min}$ at $37^{\circ} \mathrm{C}$ and subsequently filtered through $70 \mu \mathrm{m}$ nylon mesh, washed with staining buffer (1X PBS, $2 \%$ BCS, and $2 \mathrm{mM}$ EDTA) (all from Corning), and centrifuged at $800 \times \mathrm{g}$ for $10 \mathrm{~min}$. Erythrocyte lysis was done using homemade lysis buffer $(0.15 \mathrm{M}$ $\mathrm{NH}_{4} \mathrm{Cl}, 10 \mathrm{mM} \mathrm{KHCO}_{3}$, and $0.1 \mathrm{mM}$ EDTA, $\mathrm{pH}$ 7.4) (all from Corning).

The following antibodies were used for staining: CD3 (100214), CD11c (117318), anti-Mouse I-A/I-E (107620), NK1.1 (108716) CD45.2 (109824) (all from Biolegend, San Diego, CA, USA), CD4 (560782), CD25 (557192), CD8a (551162 A 1), CD45R (B220) (553093) (all from BD Biosciences, San Jose, CA, USA), CD366 (TIM3) (25-5870-80), CD279 (PD-1) (129985-83), CD103 (17-1031-82), Foxp3 (115773-82), CD152 (CTLA-4) (HMCD15201) (all from eBioscience/Thermofisher, Waltham, MA, USA), and live-dead discriminator 7AAD 7-Amino-AMD (129935) (Calbiochem/Millipore Sigma, Burlington, MA, USA). Fixation and permeabilization for intracellular markers including FoxP3 were performed using the FoxP3/ Transcription Factor Staining Buffer Set (eBioscience/Thermofisher). Intracellular cytokine 
staining was done using BD Biosciences Fixation/ Permeabilization kit specifications. Samples were run using a BD FACS Canto II (BD Biosciences) with DIVA software 6.1.3. Data were analyzed with FlowJo version 9.9.6.

\section{$T$ cell repertoire sequencing and analysis}

For TCR library preparation, we used the commercially available iRepertoire platform (iRepertoire, Huntsville, AL, USA) for nested amplicon arm-PCR of the CDR3 of the mouse TCR $\beta$ chains and addition of adaptors for Illumina platform sequencing. Reverse transcription of 300-500 ng of RNA was conducted with a OneStep reverse transcription and amplification kit (Qiagen, Germantown, MD, USA) according to the manufacturer's protocol. The PCR product was purified using Ampure XP magnetic beads (Agencourt/Beckman Coulter, Brea, CA, USA), and secondary amplification of the resulting product was performed (GoTaq PCR Kit, Promega, Madison, WI), allowing addition of Illumina adapter sequences (manufacturer's protocol). Libraries were purified with Ampure XP magnetic beads and sequenced using Illumina MiSeq $150 \mathrm{nt}$ paired-end read-length. The TCR CDR3 sequences were extracted from the raw sequencing data by iRepertoire.

\section{Statistical analysis}

All statistical analyses were performed using GraphPad Prism version 7.0 (San Diego, CA, USA), with the exception of repertoire data and RNA-Seq. Determination of statistical significance between group means was done using multiple unpaired independent $t$ tests with an alpha of 0.05 . For determination of significance between more than two group means, one-way or two-way ANOVA (analysis of variance) were used, correcting for multiple comparisons using Tukey's multiple comparisons test. Survival analysis was performed using the Kaplan-Meier method, and curves were compared with the Mantel-Cox logrank test.

Immune indices were calculated based on previously published work that identified transcripts whose expressions are highly correlated with the presence of a variety of immune cells in ovarian tumors. ${ }^{22}$ Nearly all of those genes are included on the NanoString Immune panel, which was used to analyze the RNA from these tumors. As such, we used the median expression of the genes correlated with each immune cell type as a relative estimate of the proportion of that cell type in the tumors.

Nanostring data were normalized using the varianceStabilizingTrans-formation() function in the DESeq2 package. The median variant stabilized expression value for each immune cell type was determined using the genes listed in Supplementary Table 1 . In order to determine a potential link between immune cell abundance and Wnt signaling, the Pearson correlation was calculated between expression of Axin 2 and the median expression of genes associated with estimated $\mathrm{T}$ cell abundance. The $\mathrm{R}$ statistical programming language was used to calculate all statistics related to the immune indices.

The TCR high-throughput sequencing data were analyzed in an $\mathrm{R}$ environment using the tcR package $^{23}$ and common $\mathrm{R}$ routines. Comparative analysis of TCR repertoire richness was performed after normalization of the high throughput sequencing depth. The total datasets were downsampled to 440,000 randomly chosen sequencing reads using bootstrapping with 100 iterations. A median of each simulated richness distribution was used and the treatment groups were compared using the nonparametric Mann-Whitney test. ${ }^{24}$

\section{Results}

\section{Expression of genes associated with $T$ cell} exclusion in human ovarian cancer ascites correlates with WNT974 response

To test the response of ovarian cancer to Wnt/ $\beta$ catenin inhibition, we cultured 53 human ovarian cancer ascites samples with WNT974 for 7 days and tested cell viability by ATPlite. Demographic information and tumor characteristics for the patient samples have been previously reported. ${ }^{25}$ Treated samples had a variable response to WNT974, ranging from 0 to $97 \%$ decrease in cell number, but only 10 of 53 samples had $\geqslant 19 \%$ cell kill. The 10 samples that had the highest response to WNT974 ( $\geqslant 19 \%$ decrease) were deemed 'responders'. The 10 samples that had the worst response to WNT974 ( $<10 \%$ decrease) were deemed 'nonresponders' (Figure 1(a)).

We extracted RNA from untreated responder and nonresponder samples and performed RNA-Seq. Comparison between the two groups revealed 187 differentially expressed genes (DEGs) that met a 

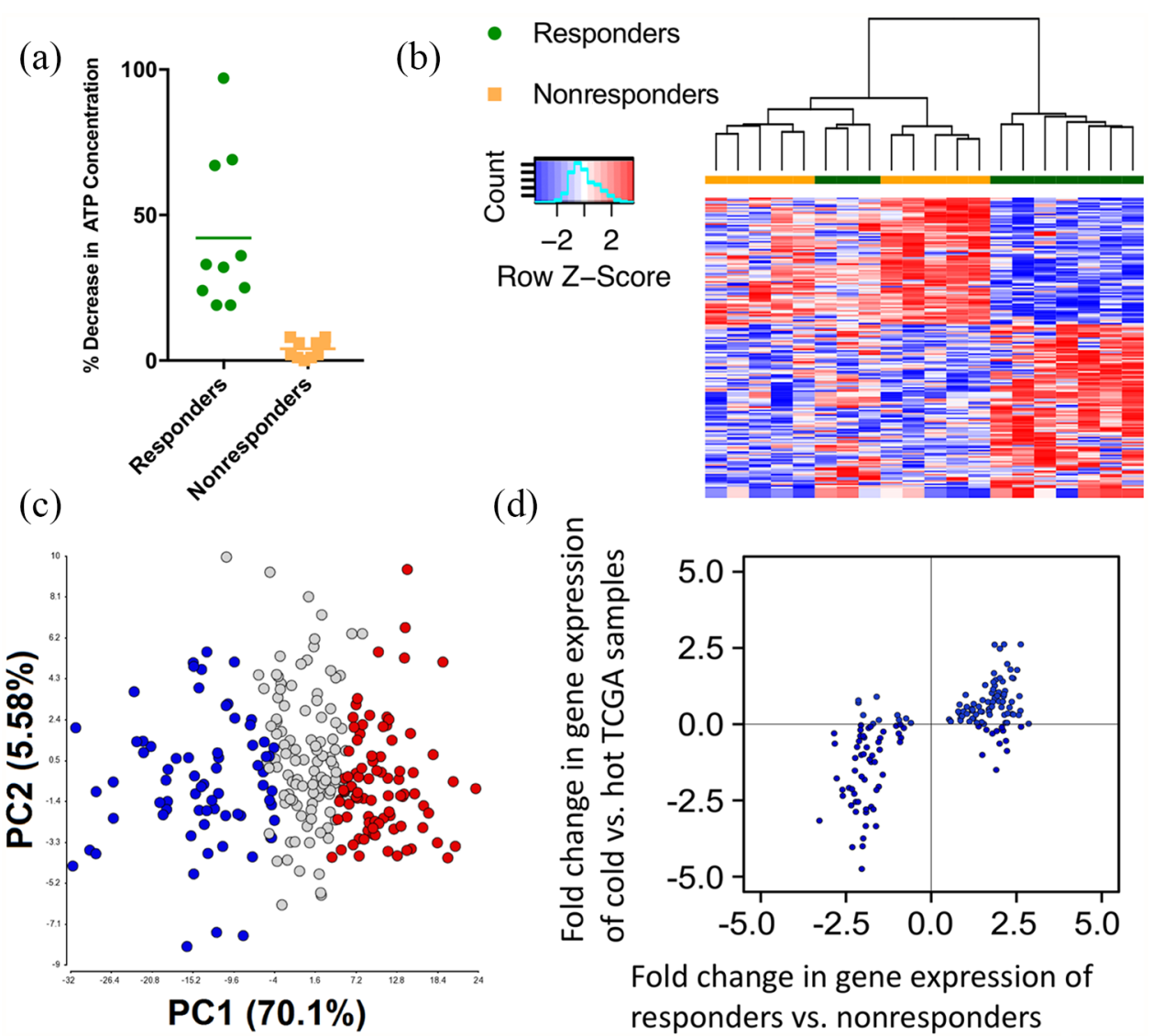

(d)

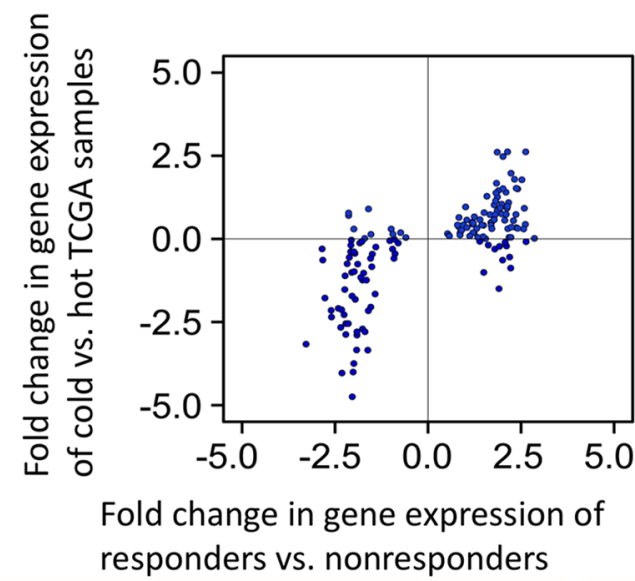

Figure 1. Effect of Wnt inhibition on human ovarian cancer samples. (a) Ascites samples ( $n=53$ ) had variable responses to 7 days of treatment with $50 \mathrm{nM}$ WNT974. Shown are samples with $\geqslant 19 \%$ decrease in ATP concentration $(n=10)$ and those that had $<10 \%$ decrease in ATP concentration $(n=10)$, which were deemed 'responders' and 'nonresponders', respectively. (b) RNA-Seq libraries of ascites samples depicted in panel (a) revealed 187 differentially expressed genes (DEGs) between responders (green) and nonresponders (yellow). (c) Principle component analysis (PCA) of 261 TCGA primary ovarian tumor samples based on the expression of a defined T-cell inflamed gene signature. The three patient groups clearly segregate by the first principle component (PC1), which explains $70.1 \%$ of the sample variance (blue=non-T-cell inflamed 'cold' group; red=T-cell inflamed 'hot' group; gray=intermediate). (d) Log2-transformed expression fold changes of 161 significant DEGs from the ascites samples treated with WNT974 (panel (b)) that were also found in the TCGA dataset. Each dot represents the fold change in the mean expression of a single gene. The majority of the genes that had a positive fold change in the WNT974 responders (relative to nonresponders) also had a positive fold change in the non-T-cell inflamed 'cold' (relative to T-cell inflamed 'hot') TCGA samples (blue samples from panel (c)). The same was true for genes that had a negative fold change.

false discovery rate cutoff of $<0.05$. Hierarchical clustering analysis of DEGs segregated samples into groups largely defined by responder and nonresponder status (Figure 1(b)).

Publicly available gene expression data of 261 ovarian serous adenocarcinoma patients were obtained from TCGA (see Methods). Using a defined $\mathrm{T}$-cell inflamed gene signature, ${ }^{19}$ which has been correlated with evidence of $\mathrm{CD}^{+} \mathrm{T}$ cell infiltration, ${ }^{26,27}$ we identified patients who had a T-cell inflamed (referred to as 'hot') or non-T-cell inflamed (referred to as 'cold') tumor microenvironment (Figure 1(c)). Among the 187 significant DEGs from the ovarian cancer ascites responder/ nonresponder groups, 161 genes were also found in the TCGA ovarian cancer dataset by matching the identical symbols. The direction of the fold change (up or down) in gene expression of these 161 genes associated with both $\mathrm{T}$ cell infiltration and WNT974 response showed a high degree of correlation (Figure 1(d)). These data led us to seek further evidence that active Wnt signaling contributes to $\mathrm{T}$ cell exclusion from the ovarian cancer 


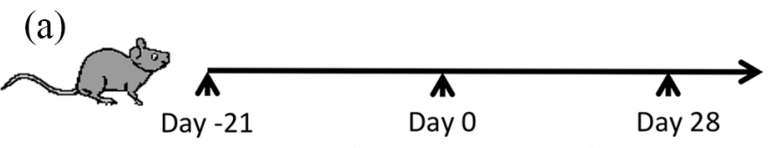

SC Tumor injection Start treatment Stop treatment

(b) $\rightarrow$ Vehicle Control

(b) $\rightarrow$ WNT974

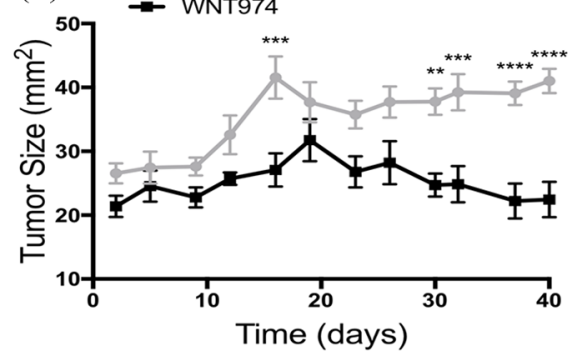

(c)

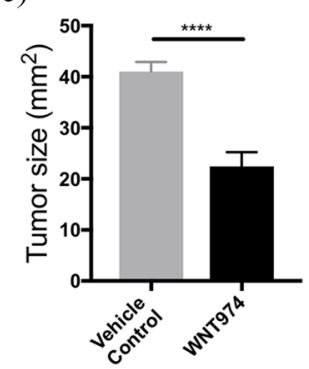

(1)
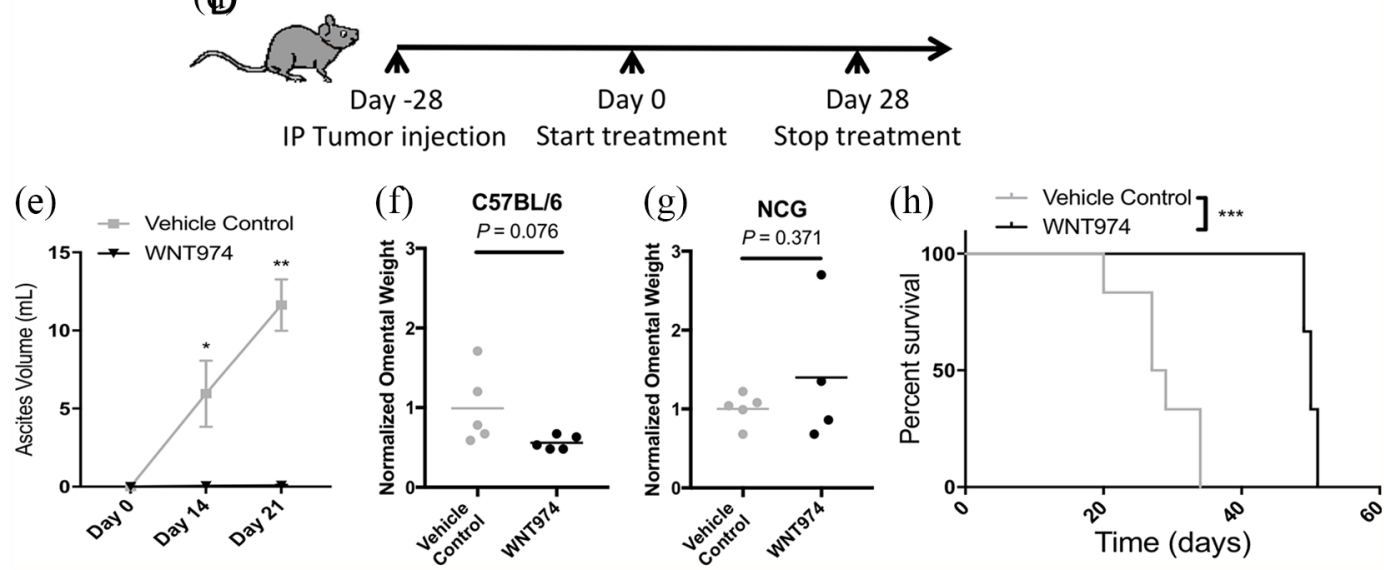

Figure 2. Effect of Wnt inhibition on tumor growth, ascites formation, and survival. (a) Subcutaneous (SC) experimental design. (b) Mice treated with WNT974 had decreased SC tumor growth compared to controls. (c) Average SC tumor size at day 40 was significantly smaller in WNT974-treated mice $(n=7$ mice/group, data from one of two independent experiments). (d) Intraperitoneal (IP) experimental design. (e) WNT974-treated mice with IP tumors had fewer ascites than control mice $(n=5-7$ mice/group, data from one of three independent experiments). (f) C57BL/6 mice treated with WNT974 had lower omental weights than control mice ( $n=5$ mice/ group). (g) NCG mice treated with WNT974 had similar omental weights to control mice ( $n=5$ mice/group). (h) WNT974-treated mice with IP tumors had prolonged survival compared to control mice ( $n=6$ mice/group). ${ }^{*} p<0.05 ;{ }^{* *} p<0.01 ;{ }^{* * *} p<0.001 ;{ }^{* * * *} p<0.0001$.

tumor microenvironment and that immunologically 'cold' tumors may be more responsive to Wnt inhibitors.

\section{Wnt inhibition reduces tumor burden and} ascites formation and prolongs survival in vivo Based on the correlative data above, we tested the ability of Wnt inhibition to impair ovarian cancer tumor growth in vivo. ID8 murine ovarian cancer cells were implanted subcutaneously into the right flank of C57BL/6 mice and allowed to grow for 21 days before being treated with WNT974 or vehicle control by oral gavage (Figure 2(a)). Mice that were treated with WNT974 had significantly reduced tumor burden compared to controls (Figure 2(b,c)). Tumors were excised from three mice per group (two at day 7 , one at day 14) and RNA expression levels were measured using the NanoString nCounter system. The $\mathrm{T}$ cell gene infiltration signature was higher in treated mice compared to controls (Supplementary Figure 1), suggesting Wnt inhibition can increase infiltration of $T$ cells into the tumor mass.

To test the effects of Wnt inhibition in an environment that more closely mimics human ovarian cancer, ID8 cells were injected IP into C57BL/6 mice, allowed to grow for 28 days, and treated with WNT974 or vehicle control (Figure 2(d)). 
(a) Gated on live $\mathrm{CD}^{+}$cells Gated on live $\mathrm{CD} 3^{+} \mathrm{CD} 4^{+}$cells

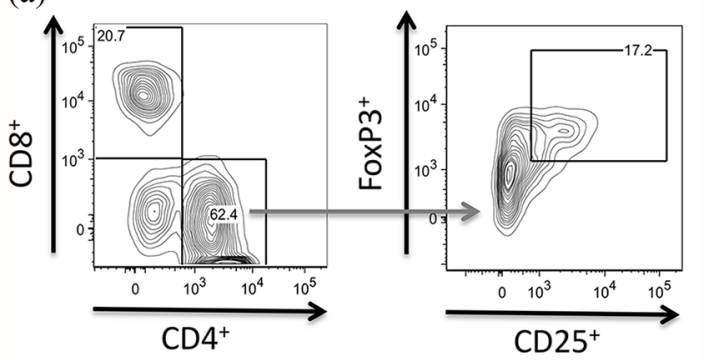

(b)

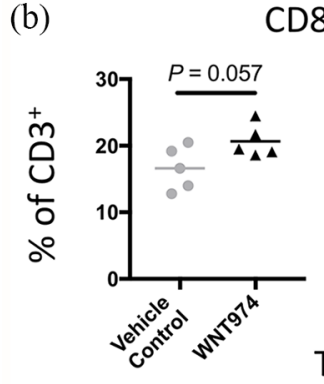

CD8 ${ }^{+} \mathrm{T}$ cells
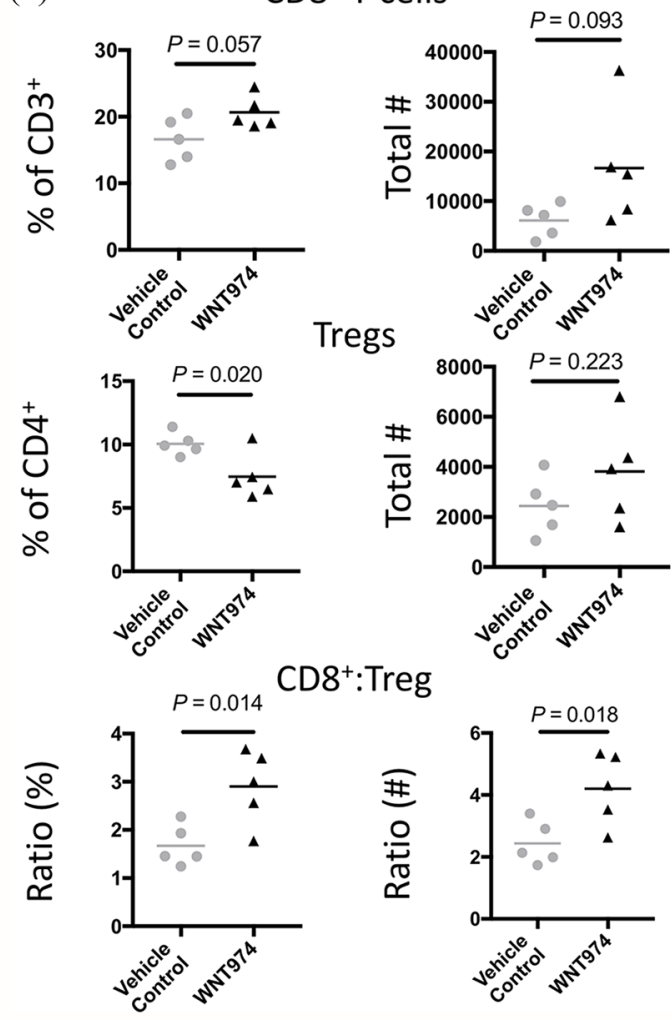

$\mathrm{CD}^{+}:$Treg

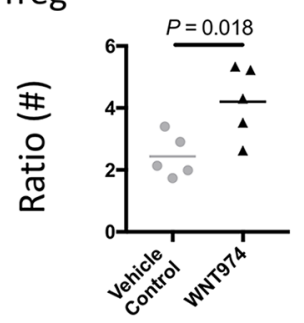

Figure 3. $\mathrm{CD}^{+} \mathrm{T}$ cell and Treg populations in omental tumor with or without treatment with WNT974 for 14 days. (a) Representative plots show gating for $\mathrm{CD}^{+}$and $\mathrm{CD} 8^{+} \mathrm{T}$ cells (left) or $\mathrm{CD}^{+}{ }^{+} \mathrm{CD} 25^{+} \mathrm{FoxP}^{+}$Tregs (right). (b) $\mathrm{CD} 8^{+} \mathrm{T}$ cells, Tregs, and $\mathrm{CD}^{+}$:Treg ratio of omental tumors. Percentage frequency of parent shown on the left, total number of cells shown on the right $(n=5-7$ mice/group, data from one of three independent experiments, dots represent individual mice).

We found that WNT974-treated mice had significantly less ascites compared to controls (Figure 2(e)). As most of the solid tumor was confined to the omentum, omental weights were compared between groups as well. There was a relative decrease in mean omental weight with 14 days of WNT974 treatment as compared to vehicle control (Figure 2(f)). To determine whether the effects of WNT974 were dependent on adaptive immunity, we used NCG mice that lack T, B, and NK cells and found that these immunodeficient mice did not have a statistical difference in their omental weights after treatment with 14 days of WNT974 (Figure 2(g)). Additionally, WNT974-treated immunocompetent mice had significantly prolonged survival compared to control mice (Figure 2(h)). Our data show that Wnt inhibition does have antitumor activity in this model and suggests that this is dependent on a functional immune system.

\section{Wnt inhibition enhances antitumor immunity}

To determine whether WNT974 altered T cell infiltration, we treated tumor-bearing mice beginning 28 days after tumor injection with either WNT974 or vehicle control and, on day 14 of treatment, evaluated $\mathrm{CD}^{+}$and $\mathrm{CD}^{+}$TILs (tumor-infiltrating lymphocytes) in the omentum (Figure 3(a)). We found that WNT974 caused a trending increase in the frequency and absolute number of intratumoral $\mathrm{CD}^{+} \mathrm{T}$ cells. Additionally, there was a decrease in the percentage frequency of Tregs of all $\mathrm{CD}_{4}^{+} \mathrm{T}$ cells, and an overall increase in the $\mathrm{CD} 8^{+}:$Treg ratio (Figure $3(b)$ ). These findings suggest that treatment with WNT974 may increase T cell infiltration into ovarian tumors, while also increasing the $\mathrm{CD}^{+}$:Treg ratio, both of which correlate with enhanced tumor control. ${ }^{28}$

Within the tumor microenvironment, many tumor-driven suppressive mechanisms limit the ability of effector $\mathrm{T}$ cells to mount an effective antitumor response. Indeed, $\mathrm{T}$ cells in the tumor microenvironment receive repetitive TCR signaling alongside various suppressive signals, eventually driving a dysfunctional $\mathrm{T}$ cell state and $\mathrm{T}$ cell exhaustion, characterized by loss of effector function and accumulation of inhibitory surface molecules. ${ }^{29}$ This led us to investigate the quality of infiltrating $\mathrm{T}$ cells by measuring cytokine production. Specifically, the activity of $\mathrm{CD} 4^{+} \mathrm{T}$ cells was assessed by production of interferon- $\gamma(\mathrm{IFN} \gamma)$ and tumor necrosis factor- $\alpha(\mathrm{TNF} \alpha)$, and that of $\mathrm{CD}^{+} \mathrm{T}$ cells by IFN $\gamma, \mathrm{TNF} \alpha$, and granzyme-B (GZB) production. We found that mice treated with WNT974 had dramatically increased cytokine production in both $\mathrm{CD} 4^{+}$and $\mathrm{CD} 8^{+} \mathrm{T}$ cell populations, suggesting much more functional TIL populations in WNT974-treated tumors (Figure 4(a)-(b)). 
(a)

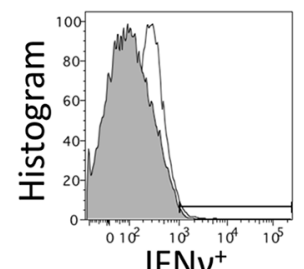

$\mathrm{CD}^{+}$

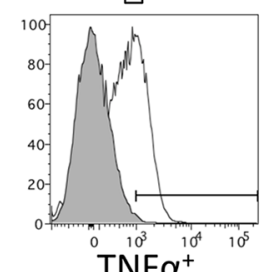

$\mathrm{CD}^{+}$
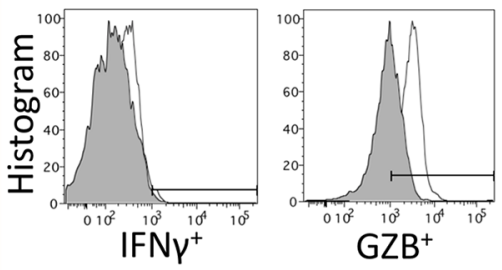

Vehicle Control WNT974

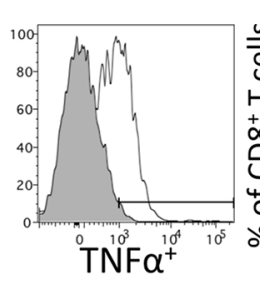

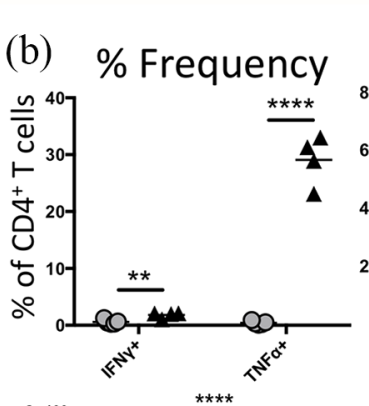
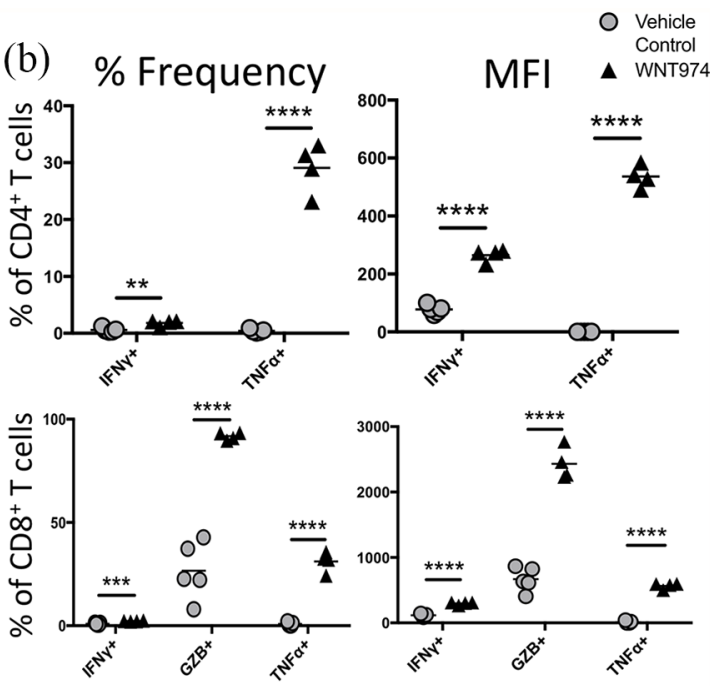

(c) Vehicle Control

WNT974

Vehicle Control

WNT974
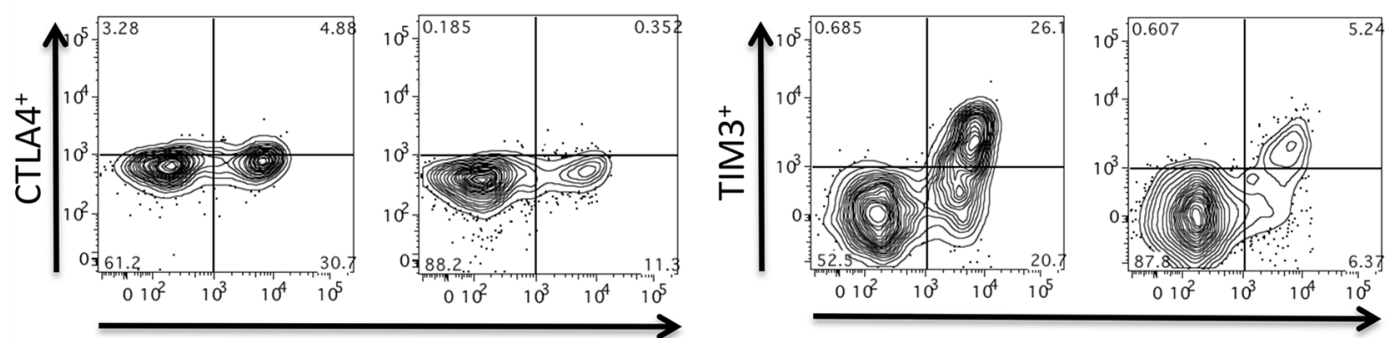

PD-1 $1^{+}$

(d)
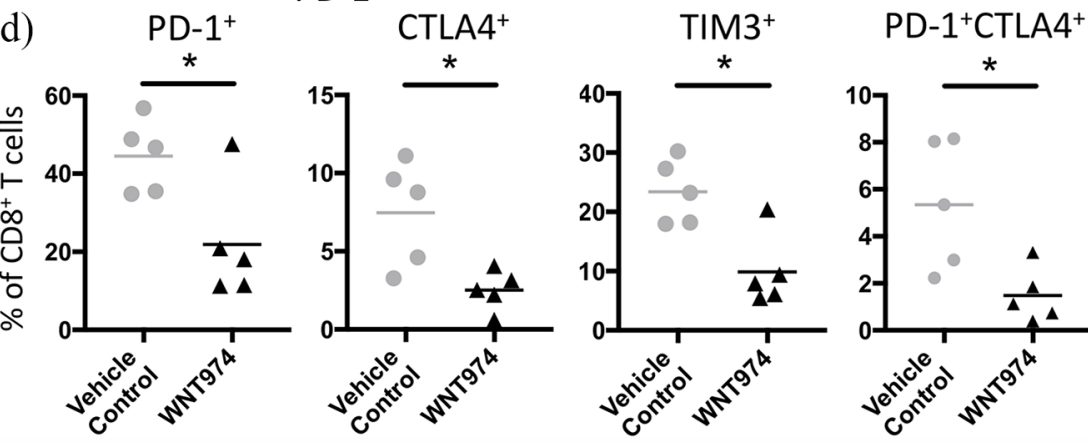

$\mathrm{PD}-1^{+}$

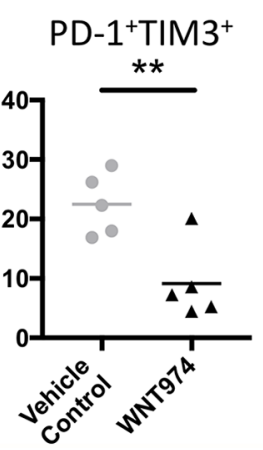

Figure 4. Cytokine and inhibitory marker expression from omental tumor TILs after 14 days of treatment. (a) Representative histograms show $\mathrm{CD}_{4}^{+} \mathrm{T}$ cells expressing IFN $\gamma$ or TNF $\alpha$ (top), $\mathrm{CD} 8^{+} \mathrm{T}$ cells expressing IFN $\gamma$, GZB, or TNF $\alpha$ (bottom). (b) Graphical representation of data from histograms shown in panel (a). $\mathrm{CD}^{+} \mathrm{T}$ cells (top) and $\mathrm{CD}^{+} \mathrm{T}$ cells (bottom), percentage frequency (left) and mean fluorescence intensity (MFI) (right) ( $n=5$ mice/group, data from one of two independent experiments). (c) Representative plots showing PD-1 and CTLA4 expression (left) and PD-1 and TIM3 expression (right) of CD8 ${ }^{+}$T cells after treatment with vehicle control or WNT974. (d) Graphical representation of data from plots shown in panel (c). Percentage frequency of CD8 $8^{+}$ cells expressing inhibitory markers ( $n=5$ mice/group, data from one of two independent experiments, dots represent individual mice).

${ }^{*} p<0.05 ;{ }^{* *} p<0.01 ;{ }^{* * *} p<0.001 ;{ }^{* * * *} p<0.0001$.

We next evaluated the expression of the inhibitory receptors PD-1, CTLA-4, and TIM3 on $\mathrm{CD}^{+} \mathrm{T}$ cells after WNT974 treatment. We observed that $\mathrm{CD}^{+} \mathrm{T}$ cells from WNT974treated tumors had decreased expression of all three markers. Furthermore, PD $-1^{+}$CTLA $-4^{+}$ and $\mathrm{PD}-1^{+}{ }^{+} \mathrm{TIM}^{+}{ }^{+}$double-positive $\mathrm{CD}^{+}{ }^{+} \mathrm{T}$ cells were less abundant after treatment with WNT974 (Figure $4(\mathrm{c}, \mathrm{d}))$. Taken together, these data suggest that Wnt inhibition increases the ratio of 


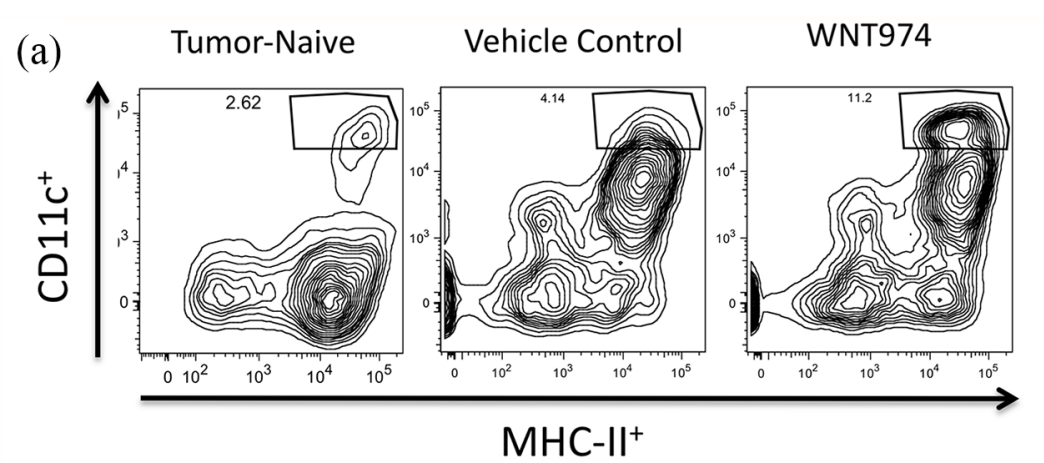

(b)

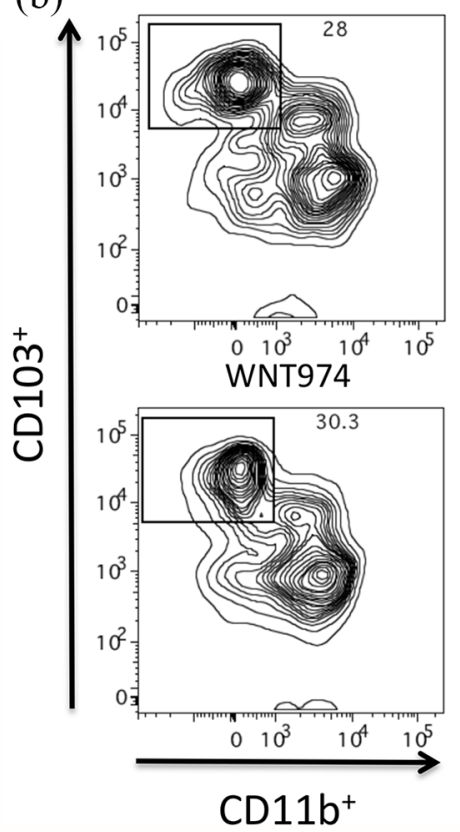

(c)

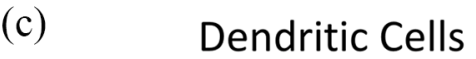

- Vehicle

Control

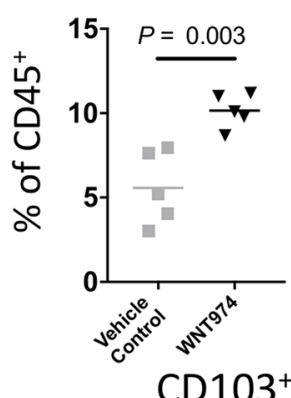

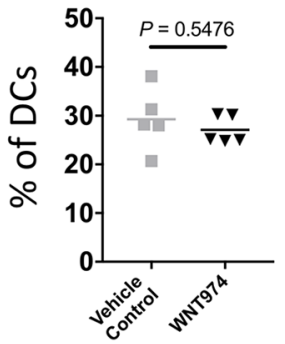

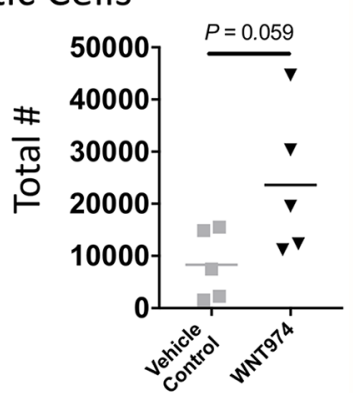

Dendritic Cells

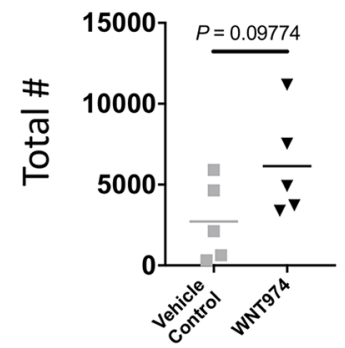

Figure 5. Dendritic cell (DC) populations in omental tumors. (a) Representative gating for MHC-II+CD11 $\mathrm{c}^{\text {hi }} \mathrm{DC}$ populations in tumor-naive or tumor-bearing mice with treatment with vehicle control or WNT974 for 14 days. (b) Representative gating for $\mathrm{CD}_{103}+\mathrm{DC}$ treated with vehicle control (top) or WNT974 (bottom). (c) Graphical representation data from plots in panels (b) and (c). Percentage frequency of parent shown on the left, total number of cells shown on the right $(n=5-7$ mice/group, data from one of three independent experiments, dots represent individual mice).

intratumoral $\mathrm{CD} 8^{+} \mathrm{T}$ cells to Tregs and increases effector activity of both $\mathrm{CD} 4^{+}$and $\mathrm{CD} 8^{+} \mathrm{T}$ cells.

Given prior evidence that the Wnt pathway prevents recruitment of $\mathrm{T}$ cells into the tumor microenvironment in melanoma through dysfunctional antigen processing in $\mathrm{CD}_{103}{ }^{+} \mathrm{DCs}$, we next investigated its effects on DCs. ${ }^{14}$ We discovered that WNT974 increased the total intratumoral DC population (Figure 5(a)), but the increase in CD103+ DCs did not reach statistical significance (Figure $5(b, c)$ ).
Wnt inhibition promotes an antitumor gene expression pattern

To better appreciate gene expression changes in the tumor microenvironment following Wnt inhibition, we treated tumor-bearing mice with WNT974 or vehicle control, and on day 14 we harvested omental tumors and analyzed mRNA expression using the PanCan Immune and PanCan Pathways NanoString probe sets. mRNA expression of target genes in the Wnt pathway, including Axin2, Fzd7, Apc, and Ctnnb1, all had significantly decreased expression after treatment 
(a)

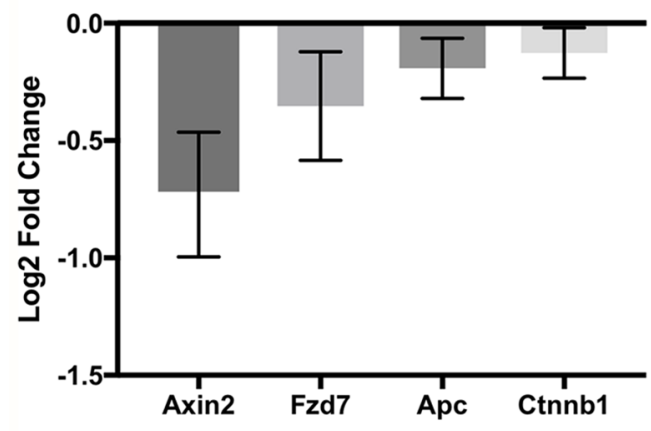

(b)

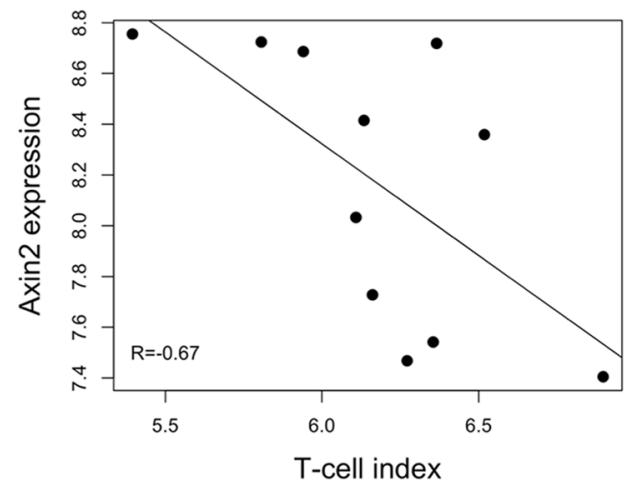

(c)

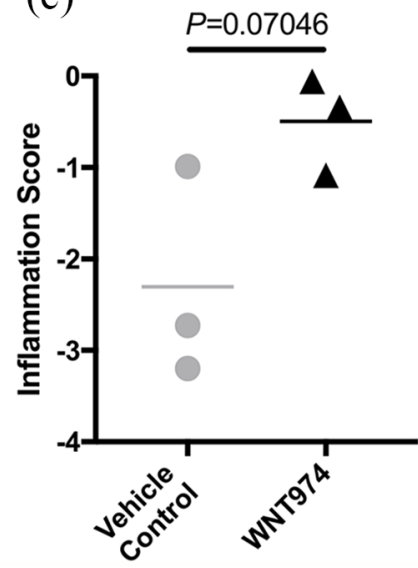

(d)

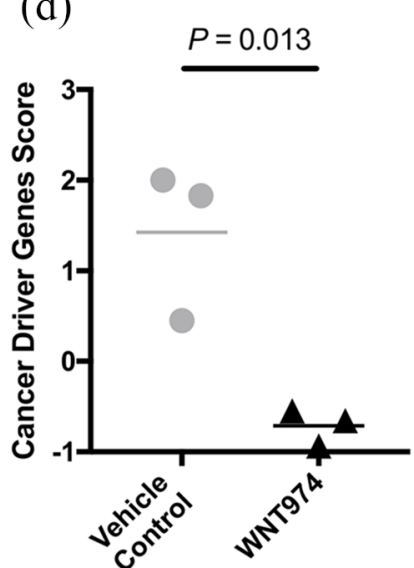

(e)

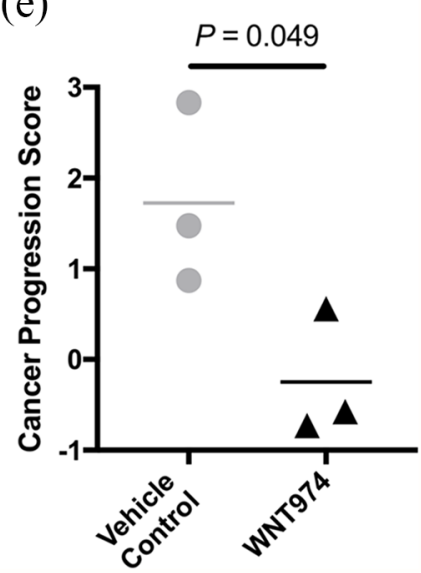

Figure 6. mRNA expression patterns from omental tumors comparing treatment with 14 days of WNT974 versus vehicle control. (a) Log2 fold change of target genes in the Wnt pathway after treatment with WNT974. (b) Gene expression of Axin2 negatively correlates with T cell infiltration. (c) NanoString-defined inflammation score. (d) NanoString-defined cancer driver genes score. (e) NanoString-defined cancer progression score. $n=3$ mice/group for each panel. See Supplementary Tables 2-4 for a full list of genes in each score.

with WNT974 compared to control mice (Figure 6(a)). In order to determine a potential link between immune cell abundance and Wnt signaling, the Pearson correlation was calculated between expression of Axin 2 and the median expression of genes associated with estimated $\mathrm{T}$ cell abundance. Axin2 expression was negatively correlated with the gene expression profile of $\mathrm{T}$ cell abundance (Figure 6(b)). The nSolver software was used to determine gene expression scores based on proprietary algorithms designed to help understand coordinated changes in the expression of multiple genes in key biological pathways. Three of those scores were analyzed to further evaluate the effect of WNT974 on omental tumors. The 'inflammation score', which reflects the expression of 113 genes involved in inflammatory pathways, was increased in treated mice (Figure 6(c)). Similarly, a 'cancer driver genes score', made up of 119 genes, and a 'cancer progression score', made up of 50 genes, were both reduced after treatment with WNT974 (Figure $6(\mathrm{~d}, \mathrm{e})$ ). These results suggest that suppression of the $\mathrm{Wnt} / \beta$-catenin pathway with WNT974 promotes inflammation and $\mathrm{T}$ cell infiltration while simultaneously suppressing oncogenic gene expression. Full lists of genes included in each analysis are available in Supplementary Tables $2-4$.

\section{Wnt inhibition combined with paclitaxel further enhances antitumor effects}

Preclinical studies show that combining paclitaxel and $\mathrm{Wnt} / \beta$-catenin inhibition is beneficial compared to either alone. ${ }^{30-32}$ In addition, the use of dose-dense paclitaxel increases $\mathrm{CD}^{+}$TILs by sparing the immune system from the severe toxicity associated with maximum tolerated dose paclitaxel. ${ }^{21}$ To test whether the combination of 
(a)

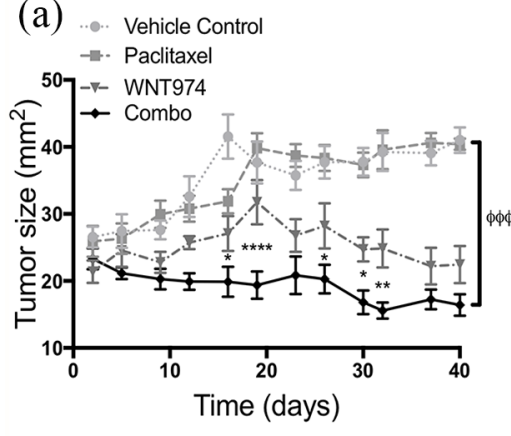

(d)

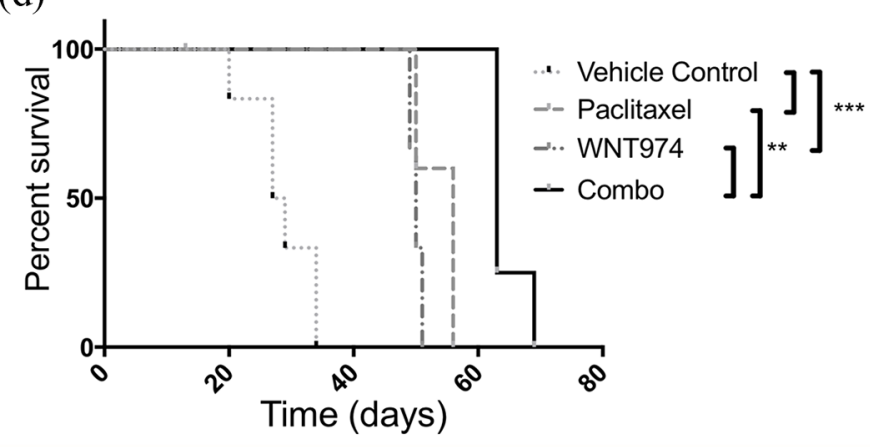

(b)

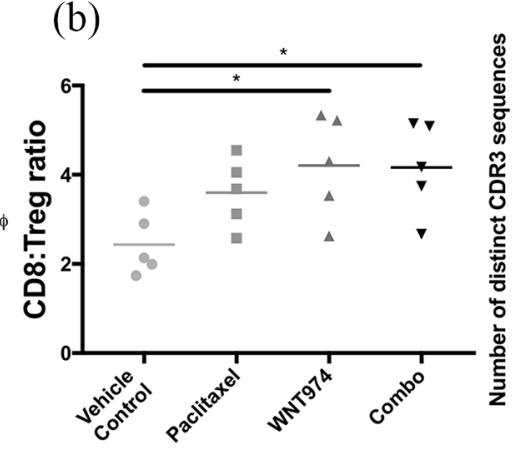

(c)

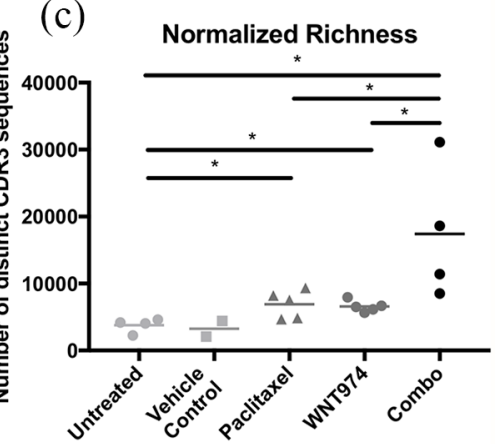

(e)

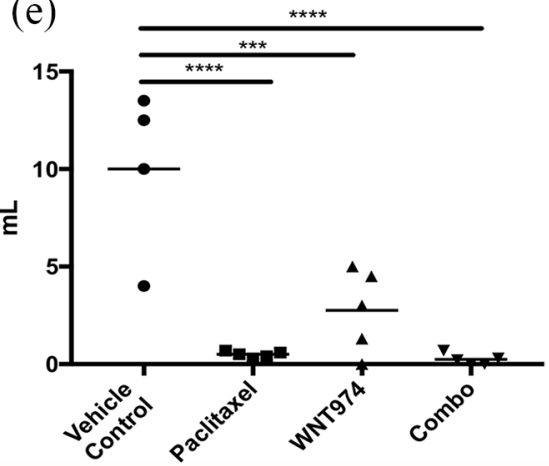

Figure 7. Combination of paclitaxel with Wnt inhibition. (a) Subcutaneous (SC) tumor growth ( $n=7$ mice/group, data from one of two independent experiments). ${ }^{*}$ indicates significance between WNT974 and Combo groups. $\phi$ indicates significance between vehicle control/paclitaxel and combo groups at endpoint. Treatment was initiated 21 days after SC tumor implantation. (b) $C D 8^{+}$:Treg ratio of omental tumors after 14 days of treatment ( $n=5-7$ mice/group, data from one of three independent experiments). Treatment was initiated 28 days after intraperitoneal (IP) tumor injection. (c) $\beta$-TCR repertoire analysis from whole peritoneal tumors after 26 days of treatment ( $n=2-5$ mice/group). (d) Survival curves of mice with IP tumors ( $n=6$ mice/group). (e) MISIIRTAg-Low mice injected IP with MISIIR ovarian cancer (MOVCAR) cells and treated with 14 days of paclitaxel, WNT974, or combination all have fewer ascites than vehicle control ( $n=5$ mice/group).

${ }^{*} p<0.05 ;{ }^{* * *} p<0.01 ;{ }^{* * *} p<0.001 ;{ }^{* * * *} p<0.0001$.

WNT974 and dose-dense paclitaxel increased immunity and decreased tumor growth, we administered dose-dense paclitaxel (3 days on/3 days off, for nine total doses) and/or WNT974 in mice with either SC or IP tumors. We found that SC tumor growth was not affected by paclitaxel alone, was reduced by WNT974 alone, and that combination treatment had the most profound effect on tumor size (Figure 7(a); Supplementary Figure 3).

In mice with IP tumors, WNT974 alone and in combination with paclitaxel increased the CD8: Treg ratio after 14 days of treatment (Figure 7(b)). Moreover, both WNT974 and paclitaxel increased the number of distinct CDR3 sequences in tumors, a difference that was more impressive when the two agents were combined (Figure 7 (c)). Finally, 28 days of treatment with both WNT974 and paclitaxel prolonged survival.
However, combination treatment resulted in significantly longer survival compared to both individual treatment arms (Figure $7(\mathrm{~d})$ ). These results are in accordance with previous reports of the benefit of combining Wnt inhibition with taxane therapies, further supporting investigation into this combination approach in clinical trials.

In order to determine whether this effect was persistent in a different mouse model, we used mice that have heterozygous expression of the Simian Virus $40 \mathrm{~T}$ Antigen (SV40-TAg) under the control of the Mullerian Inhibitory Substance Type II Receptor promoter (MISIIR-TAg-Low mice). Unlike their homozygous counterpart (MISIIRTAg mice), these mice do not spontaneously develop ovarian carcinoma, but will develop tumors if injected IP with MOVCAR cells derived from MISIIR-TAg mice. We performed IP injections of MOVCAR cells into MISIIR-TAg-Low 
mice and treated them with vehicle control, paclitaxel, WNT974, or combination for 14 days. All treatment arms had significantly less ascites compared to the control arm, similar to the results seen in the ID8 model (Figure 7(e)).

\section{Discussion}

Our initial genomic analysis showed a correlation between Wnt signaling and $\mathrm{T}$ cell infiltration in ovarian tumors. Our in vitro work suggests that response to WNT974 is variable among patientderived ascites samples and that those samples with improved response have gene expression patterns similar to those in 'immunologically cold' ovarian tumors that lack $\mathrm{T}$ cell infiltration, suggesting that the Wnt signaling pathway may promote immune evasion. In mouse models of ovarian cancer, we find that WNT974 slows tumor growth, prevents ascites, and prolongs survival - effects that are dependent on adaptive immunity. Consistent with this conclusion, WNT974 increases the expression of effector cytokines and decreases expression of inhibitory receptors by tumor-infiltrating $\mathrm{CD} 8^{+} \mathrm{T}$ cells. As expected, treatment with WNT974 decreases the expression of Wnt-dependent genes and increases the expression of genes associated with $\mathrm{T}$ cell infiltration. Taken together, these data suggest that inhibition of the Wnt signaling pathway alters the tumor microenvironment to favor antitumor immunity and impair tumor progression.

Although the $\mathrm{Wnt} / \beta$-catenin signaling pathway is clearly involved in tumorigenesis in a variety of cancer types, ${ }^{33-36}$ recent data suggest that it may also suppress antitumor immune responses. For example, active $\mathrm{Wnt} / \beta$-catenin signaling in melanoma impairs the expression of chemokines that normally recruit $\mathrm{CD} 103^{+} \mathrm{DCs}$ to the tumor. ${ }^{14} \mathrm{As}$ a result, tumors with active Wnt signaling have fewer $\mathrm{CD}_{103}{ }^{+} \mathrm{DCs}$ and fail to activate local $\mathrm{CD} 8{ }^{+} \mathrm{T}$ cell responses. ${ }^{14}$ Our data suggest that a similar phenomenon occurs in ovarian cancer. While we do not see a significant increase in CD103+ DCs in tumors from WNT974-treated mice, we do observe dramatic increases in the activity of tumor-infiltrating $\mathrm{CD}^{+} \mathrm{T}$ cells changes that correspond with decreases in tumor volume and prolonged survival. Wnt-related immunosuppression has been associated with lower expression of chemokines CCL3, CCL4, CXCL1, and CXCL2. ${ }^{14}$ In our model, expression of these chemokines by omental tumor after treatment with WNT974 as measured by
NanoString showed mixed responses, with decreased expression of CCL3 and CCL4, but increased expression of CXCL1 and CXCL2 (Supplementary Figure 2). Nonetheless, our data support findings that the Wnt signaling pathway suppresses antitumor immunity in ovarian cancer and can be pharmacologically targeted to reverse the effect. Our results indicate that this is likely due to an upstream effect on tumor $\mathrm{T}$ cell infiltration as opposed to activating mutations in the $\mathrm{Wnt} / \beta$-catenin pathway, and is in accordance with other recent publications. ${ }^{37-39}$

Although Wnt inhibitors may act on tumor cells to indirectly enhance the immune responses, these same inhibitors may also directly impact the activity of immune cells. For example, Wnt signaling in Tregs activates TCF-1, which competes with FoxP3 for binding to a variety of genes that are important for their suppressive activity. ${ }^{40}$ Similarly, the activation of TCF-1 in Tregs promotes their conversion to inflammatory Th17 cells. ${ }^{41}$ Thus, by inhibiting Wnt signaling, Tregs may become more suppressive and actually impair antitumor immunity.

Wnt also acts on $\mathrm{CD}^{+} \mathrm{T}$ cells. For example, the Wnt-mediated activation of Tcf- 1 in $\mathrm{CD}^{+} \mathrm{T}$ cells promotes their differentiation into stem celllike memory $\mathrm{T}$ cells ${ }^{42}$ and counteracts $\mathrm{T}$ cell exhaustion. ${ }^{43}$ These functions are likely related to the ability of Tcf-1 to counteract IL-2-mediated expression of BLIMP-1. ${ }^{44}$ As a result, the inhibition of Wnt signaling would likely decrease Tcf-1 activity and promote the differentiation of effector $\mathrm{T}$ cells. In fact, this type of result would be consistent with our observation that WNT974 increases the production of IFN $\gamma, \mathrm{TNF}$, and granzyme-B by $\mathrm{CD}^{+} \mathrm{T}$ cells. Although the expression of immune checkpoints on $\mathrm{T}$ cells is lower with WNT974 treatment, it is still present and therefore the addition of immune checkpoint inhibitors may result in an even more robust antitumor immune response. Further evaluation of this therapeutic combination is a compelling next step.

WNT974 may also be acting directly on DCs. $\beta$ catenin signaling in DCs is important for maintaining their tolerogenic ability. ${ }^{13}$ In the absence of $\beta$-catenin, DCs become activated in the absence of microbial stimuli and promote $\mathrm{T}$ cell activation and autoimmunity. ${ }^{13}$ In some instances, $\beta$-catenin can be activated in DCs via mechanical, non-Wnt-mediated pathways, ${ }^{45}$ which still 
lead to the differentiation of tolerogenic DCs. However, if Wnt inhibitors block $\beta$-catenin activity in DCs, they are likely to be more immunogenic and more efficiently prime tumor-reactive $\mathrm{T}$ cells, again consistent with our results. In addition to its effects on DCs and CD8 ${ }^{+} \mathrm{T}$ cells, Wnt inhibition may modulate the activity of several other leukocyte subsets that are involved in the antitumor immune response in this model.

Our data also show that the combination of WNT974 and paclitaxel further enhances immune parameters and decreases tumor growth better than either agent alone. The effectiveness of this combination is likely due to the overlap in the mechanism of action of paclitaxel, which stabilizes microtubules, and $\beta$-catenin, which stabilizes mitotic spindles and facilitates centrosome separation. ${ }^{46-48}$ Thus, the combined effects of microtubule stabilization and Wnt inhibition can dramatically inhibit proliferation and promote tumor cell death. Importantly, treatment with taxane alone may select for cells that can overcome microtubule stabilization through mechanisms that require increased Wnt pathway activity. ${ }^{30}$ Consequently, Wnt pathway inhibitors may be particularly useful in the prevention of chemoresistance. In addition, combination treatment couples the immune benefits of a more active $\mathrm{CD} 8^{+} \mathrm{T}$ cell response from Wnt inhibition with an increased TIL presence offered by dosedense taxol administration. Therefore, in tumor types that are known to have highly active $\mathrm{Wnt} / \beta$ catenin activity, such as colon and endometrial cancers, Wnt inhibition may serve a dual purpose, with both immunostimulatory and direct antitumor effects. Limiting factors to implementation of this strategy are related to concerns about the toxicity of WNT974, particularly on cells that rely on the Wnt pathway to maintain homeostasis, including intestinal epithelium, osteoclasts, and osteoblasts. An ongoing phase I trial of oral WNT974 [ClinicalTrials.gov identifier: NCT01351103] is currently enrolling to further assess these issues.

Our experiments had several limitations. First, our ID8 cell line has an intact $p 53$ gene, which is consistently mutated in human ovarian cancer and may affect the behavior of these cells in vitro and in vivo. Second, due to the toxicity of the chemotherapeutic drugs that were used, there was some variation in the numbers of mice between experiments (numbers per group are indicated throughout the figure legends). Third, paclitaxel alone did not have a significant effect on SC tumor growth. We think this is partially due to the IP (as opposed to IV) administration of paclitaxel, but suspect that our data may also have suffered from the relatively slow growth of the SC tumors causing differences between groups to be more difficult to interpret. Finally, although inhibition of the Wnt/ $\beta$-catenin pathway appears to have a significant effect on the immune response to ovarian cancer in these models, applicability of our results to clinical practice is purely speculative at this time. Further experimentation in human ovarian cancer patients is required to elicit the appropriate role of Wnt $/ \beta$-catenin blockade in the treatment of ovarian cancer. While our data suggest that an intact immune system is required for Wnt/ $\beta$-catenin inhibition to be fully effective, further experimentation is required to completely elucidate its role.

Taken together, our data suggest that inhibiting the Wnt signaling pathway in ovarian cancer impairs the tumorigenic activity of tumor cells and increases their inflammatory properties, and at the same time promotes antitumor immunity, probably by a combination of effects on tumor cells and immune cells. Interestingly, in human cancer cells, inhibition of WNT974 appears to be most effective in the samples exhibiting a 'cold' immune signature. This study has important implications for therapy of 'cold' ovarian cancers, as these in general carry an inferior prognosis. With increasing emphasis on the importance of antitumor immunity in cancer treatment, blockade of the Wnt/ $\beta$-catenin pathway should not be overlooked as a potential target in the treatment of human ovarian malignancies.

\section{Funding}

The authors disclosed receipt of the following financial support for the research, authorship, and/or publication of this article: The Norma Livingston Ovarian Cancer Foundation, ABOG/ AAOGF, UAB Cancer Center, UAB Center for Clinical and Translational Science, Foundation for Women's Cancer, American Cancer Society (grant number IRG-60-001-53-IRG), NIH (grant number RO1-CA216234 to TDR and T32-CA183926 Research Training Program in Basic and Translational Oncology to AIL), and Tie the Ribbons Fund.

\section{Conflict of interest statement}

$\mathrm{JJL}$ is a consultant to and receives clinical trial research support from Novartis. 
The remaining authors declare no potential conflicts of interest.

\section{ORCID iD}

Ilaria Betella (iD https://orcid.org/0000-00019191-8228

\section{Supplemental material}

Supplemental material for this article is available online.

\section{References}

1. Siegel RL, Miller KD and Jemal A. Cancer statistics, 2018. CA Cancer f Clin 2018; 68: $7-30$.

2. Wu G, Liu A, Zhu J, et al. MiR-1207 overexpression promotes cancer stem cell-like traits in ovarian cancer by activating the Wnt/ beta-catenin signaling pathway. Oncotarget 2015; 6: 28882-28894.

3. Bodnar L, Stanczak A, Cierniak S, et al. Wnt/ beta-catenin pathway as a potential prognostic and predictive marker in patients with advanced ovarian cancer. $\mathcal{F}$ Ovarian Res 2014; 7: 16.

4. Polakis P. Wnt signaling in cancer. Cold Spring Harb Perspect Biol 2012; 4: pii: a008052.

5. Takebe N, Harris PJ, Warren RQ, et al. Targeting cancer stem cells by inhibiting Wnt, Notch, and Hedgehog pathways. Nat Rev Clin Oncol 2011; 8: 97-106.

6. Verras $M$ and Sun Z. Roles and regulation of Wnt signaling and $\beta$-catenin in prostate cancer. Cancer Lett 2006; 237: 22-32.

7. Talbot LJ, Bhattacharya SD and Kuo PC. Epithelial-mesenchymal transition, the tumor microenvironment, and metastatic behavior of epithelial malignancies. Int F Biochem Mol Biol 2012; 3: 117-136.

8. Clevers $\mathrm{H}$ and Nusse $\mathrm{R}$. Wnt/ $\beta$-catenin signaling and disease. Cell 2012; 149: 1192-1205.

9. MacDonald BT and He X. Frizzled and LRP5/6 receptors for Wnt/ $\beta$-catenin signaling. Cold Spring Harb Perspect Biol 2012; 4: a007880.

10. Miranda M, Galli LM, Enriquez M, et al. Identification of the WNT1 residues required for palmitoylation by Porcupine. FEBS Lett 2014; 588: 4815-4824.

11. Liu J, Pan S, Hsieh MH, et al. Targeting Wntdriven cancer through the inhibition of Porcupine by LGK974. Proc Natl Acad Sci U S A 2013; 110: 20224-20229.
12. Pai SG, Carneiro BA, Mota JM, et al. Wnt/ $\beta$ catenin pathway: modulating anticancer immune response. F Hematol Oncol 2017; 10: 101.

13. Manicassamy S, Reizis $\mathrm{B}$, Ravindran $\mathrm{R}$, et al. Activation of $\beta$-catenin in dendritic cells regulates immunity versus tolerance in the intestine. Science 2010; 329: 849-853.

14. Spranger S, Bao R and Gajewski TF. Melanomaintrinsic $\beta$-catenin signalling prevents anti-tumour immunity. Nature 2015; 523: 231-235.

15. Arend RC, Londono-Joshi AI, Samant RS, et al. Inhibition of Wnt $/ \beta$-catenin pathway by niclosamide: a therapeutic target for ovarian cancer. Gynecol Oncol 2014; 134: 112-120.

16. Alonso A, Lasseigne $\mathrm{BN}, \mathrm{W}$ illiams $\mathrm{K}$, et al. aRNApipe: a balanced, efficient and distributed pipeline for processing RNA-seq data in high-performance computing environments. Bioinformatics 2017; 33: 1727-1729.

17. Love MI, Huber W and Anders S. Moderated estimation of fold change and dispersion for RNA-seq data with DESeq2. Genome Biol 2014; 15: 550 .

18. Li B and Dewey CN. RSEM: accurate transcript quantification from RNA-Seq data with or without a reference genome. BMC Bioinformatics $2011 ; 12: 323$

19. Spranger S, Luke JJ, Bao R, et al. Density of immunogenic antigens does not explain the presence or absence of the T-cell-inflamed tumor microenvironment in melanoma. Proc Natl Acad Sci U S A 2016; 113: E7759-E7768.

20. Quinn BA, Xiao F, Bickel L, et al. Development of a syngeneic mouse model of epithelial ovarian cancer. F Ovarian Res 2010; 3: 24.

21. Chang CL, Hsu YT, Wu CC, et al. Dose-dense chemotherapy improves mechanisms of antitumor immune response. Cancer Res 2013; 73: 119-127.

22. Danaher P, Warren S, Lu R, et al. Pan-cancer adaptive immune resistance as defined by the tumor inflammation signature (TIS): results from the cancer genome atlas (TCGA). F Immunother Cancer 2018; 6: 63.

23. Nazarov VI, Pogorelyy MV, Komech EA, et al. tcR: an $\mathrm{R}$ package for $\mathrm{T}$ cell receptor repertoire advanced data analysis. BMC Bioinformatics 2015; 16: 175 .

24. Venturi V, Kedzierska K, Turner SJ, et al. Methods for comparing the diversity of samples of the $\mathrm{T}$ cell receptor repertoire. $\mathcal{F}$ Immunol Methods 2007; 321: 182-195.

25. Boone JD, Arend RC, Johnston BE, et al. Targeting the Wnt/ $\beta$-catenin pathway in primary 
ovarian cancer with the porcupine inhibitor WNT974. Lab Invest 2016; 96: 249-259.

26. Spranger S, Spaapen RM, Zha Y, et al. Up-regulation of PD-L1, IDO, and $T_{\text {regs }}$ in the melanoma tumor microenvironment is driven by CD8+> T cells. Sci Transl Med 2013; 5: 200 ra116.

27. Harlin H, Meng Y, Peterson AC, et al. Chemokine expression in melanoma metastases associated with CD8 + T-cell recruitment. Cancer Res 2009; 69: 3077-3085.

28. Sato E, Olson SH, Ahn J, et al. Intraepithelial $\mathrm{CD}^{+}$tumor-infiltrating lymphocytes and a high $\mathrm{CD}^{+} /$regulatory $\mathrm{T}$ cell ratio are associated with favorable prognosis in ovarian cancer. Proc Natl Acad Sci U S A 2005; 102: 18538-18543.

29. Wherry EJ, Ha SJ, Kaech SM, et al. Molecular signature of $\mathrm{CD}^{+} \mathrm{T}$ cell exhaustion during chronic viral infection. Immunity 2007; 27: 670-684.

30. Fischer MM, Cancilla B, Yeung VP, et al. WNT antagonists exhibit unique combinatorial antitumor activity with taxanes by potentiating mitotic cell death. Sci Adv 2017; 3: e1700090.

31. Flores ML, Castilla C, Gasca J, et al. Loss of PKC $\delta$ induces prostate cancer resistance to paclitaxel through activation of wnt/ $\beta$-catenin pathway and Mcl-1 accumulation. Mol Cancer Ther 2016; 15: 1713-1725.

32. Chikazawa N, Tanaka H, Tasaka T, et al. Inhibition of Wnt signaling pathway decreases chemotherapy-resistant side-population colon cancer cells. Anticancer Res 2010; 30: 2041-2048.

33. Clements WM, Wang J, Sarnaik A, et al. $\beta$ catenin mutation is a frequent cause of Wnt pathway activation in gastric cancer. Cancer Res 2002; 62: 3503-3506.

34. Satoh S, Daigo Y, Furukawa Y, et al. AXIN1 mutations in hepatocellular carcinomas, and growth suppression in cancer cells by virusmediated transfer of AXIN1. Nat Genet 2000; 24 : 245-250.

35. Dahmen RP, Koch A, Denkhaus D, et al. Deletions of AXIN1, a component of the WNT/ wingless pathway, in sporadic medulloblastomas. Cancer Res 2001; 61: 7039-7043.

Visit SAGE journals online journals.sagepub.com/ home/tam

(SAGE journals
36. Segditsas $S$ and Tomlinson I. Colorectal cancer and genetic alterations in the Wnt pathway. Oncogene 2006; 25: 7531-7537.
37. Ganesh S, Shui X, Craig KP, et al. RNAimediated $\beta$-catenin inhibition promotes $T$ cell infiltration and antitumor activity in combination with immune checkpoint blockade. Mol Ther 2018; 26: 2567-2579.

38. Rudy SF, Brenner JC, Harris JL, et al. In vivo Wnt pathway inhibition of human squamous cell carcinoma growth and metastasis in the chick chorioallantoic model. f Otolaryngol Head Neck Surg 2016; 45: 26.

39. Fernandez D, Guereno M, Lago Huvelle MA, et al. Signaling network involved in the GPC3induced inhibition of breast cancer progression: role of canonical Wnt pathway. $\mathcal{F}$ Cancer Res Clin Oncol 2018; 144: 2399-2418.

40. van Loosdregt J, Fleskens V, Tiemessen MM, et al. Canonical Wnt signaling negatively modulates regulatory $\mathrm{T}$ cell function. Immunity 2013; 39: 298-310.

41. Keerthivasan S, Aghajani K, Dose M, et al. $\beta$-catenin promotes colitis and colon cancer through imprinting of proinflammatory properties in T cells. Sci Transl Med 2014; 6: 225 ra228.

42. Gattinoni L, Zhong XS, Palmer DC, et al. Wnt signaling arrests effector $\mathrm{T}$ cell differentiation and generates $\mathrm{CD}^{+}$memory stem cells. Nat Med 2009; 15: 808-813.

43. Wu T, Ji Y, Moseman EA, et al. The TCF1Bcl6 axis counteracts type I interferon to repress exhaustion and maintain T cell stemness. Sci Immunol 2016; 1: eaai8593.

44. Wu T, Shin HM, Moseman EA, et al. TCF1 is required for the $\mathrm{T}$ follicular helper cell response to viral infection. Cell Rep 2015; 12: 2099-2110.

45. Jiang A, Bloom O, Ono S, et al. Disruption of E-cadherin-mediated adhesion induces a functionally distinct pathway of dendritic cell maturation. Immunity 2007; 27: 610-624.

46. Krieg EF Jr, Mathias PI, Toennis CA, et al. Detection of DNA damage in workers exposed to JP-8 jet fuel. Mutat Res 2012; 747: 218-227.

47. Stolz A, Neufeld K, Ertych N, et al. Wntmediated protein stabilization ensures proper mitotic microtubule assembly and chromosome segregation. EMBO Rep 2015; 16: 490-499.

48. Mbom BC, Siemers KA, Ostrowski MA, et al. Nek2 phosphorylates and stabilizes $\beta$-catenin at mitotic centrosomes downstream of Plk1. Mol Biol Cell 2014; 25: 977-991. 